\title{
Multiband Quantum Transmitting Boundary Method for Non-orthogonal Basis
}

\author{
G.-C. LIANG ${ }^{\text {a }}$, Y. A. LIN $^{\text {a }}$, D. Z.-Y. TING ${ }^{\text {a, }}$ * and Y.-C. CHANG ${ }^{\text {b }}$ \\ ${ }^{a}$ Department of Physics, National Tsing Hua University, Hsinchu, Taiwan 300, ROC, \\ ${ }^{\mathrm{b}}$ Department of Physics, University of Illinois at Urbana-Champaign $1110 \mathrm{~W}$. Green St., \\ Urbana, Illinois 61801
}

\begin{abstract}
We generalize the Multiband Quantum Transmitting Boundary Method (MQTBM) for computing transmission coefficients in heterostructure to tight-binding-like band structure models with non-orthogonal basis and multiple neighbor interactions. We implement this method based on the newly developed planar-basis pseudopotential method which uses the generalized planar Wannier function basis. We demonstrate the method by computing transmission coefficients for a GaAs/AlAs double barrier structure.
\end{abstract}

Keywords: Resonant tunneling, tight binding, non-orthogonal basis, transmission coefficient

\section{INTRODUCTION}

Since Chang, Esaki and Tsu first observed resonant tunneling effect in heterostructures in 1974 [1], many theoretical methods have been developed to model the physics of resonant tunneling phenomenon in semiconductor heterostructures. The most commonly used formalism for computing tunneling probabilities in these structures is the transfermatrix method. Unfortunately, this method is numerically unstable, and especially so for multiband models. One solution which circumvents the instability problem is the Multiband Quantum Transmitting Boundary Method (MQTBM) [2].
The MQTBM is a multiband realization of the Quantum Transmitting Boundary Method originally developed by Lent and Kirkner [3] for treating quantum transport in nanostructures. Thus far, MQTBM has been implemented for several realistic, empirical, multiband models, including the tight-binding model, the effective bond orbital model and the $\mathbf{k} \cdot \mathbf{p}$ method [4].

Recently, Chang has developed the planar-basis pseudopotential band structure model for treating electronic properties of surfaces and heterostructures $[5,6]$. For treating structures with layer-like local geometry, this model is considerably more efficient than plane-wave based pseudopotential

*Corresponding author. Tel.: +886.3.574.2518, Fax: +886.3.572.3052. E-mail: dzt@phys.nthu.edu.tw. 
methods, and leads to better analysis of surface and interface properties. Furthermore, it can be constructed directly from first principles and thus offers the possibility for more physically accurate descriptions than could be obtained with empirical band structure models. The method has been successfully applied to the studies of electronic and optical properties of surface and interfaces. We implement MQTBM for this new band structure model so that it can be used to treat quantum transport problems as well. The present work differs from MQTBM implementations in the past in that the basis functions used are non-orthogonal, and thus introduces some new complications.

\section{METHOD}

\section{A. Planar-Basis Pseudopotential Method and Planar Wannier Functions}

We briefly introduce the planar-basis pseudopotential method and planar Wannier functions, and refer the readers to the work by Chang $[5,6]$ for more details. In the planar-basis pseudopotential method, planar-basis functions consisting of $2 \mathrm{D}$ plane waves in the $x-y$ plane and 1D gaussian functions in the $z$ direction are used in heterostructure electronic calculations $[5,6]$. The method can be made formally equivalent to either empirical or self-consistent first principles pseudopotential methods. Using these basis functions, we can find Bloch states associated with band $n$ and wave vector $\boldsymbol{k}, \psi_{n, \boldsymbol{k}}(\boldsymbol{r})$ and use them in turn to construct the planar Wannier functions. Assuming the heterostructure growth direction is along the $z$ axis, a planar Wannier function is a translationally invariant function in the $x-y$ plane characterized by an in-plane wavevector $\boldsymbol{k}_{\|}$, and centered along the growth direction at $R_{z}$ :

$$
\left\langle\boldsymbol{r} \mid W_{n, \boldsymbol{k}_{\|}}\left(R_{z}\right)\right\rangle=\int d k_{z} e^{-i k_{z} R_{z}} \psi_{n, \boldsymbol{k}}(\boldsymbol{r}) .
$$

For a fixed value of $\boldsymbol{k}_{\|}$, the set of Wannier functions with same band index $n$ and centered at different $R_{z}$ may be thought of as a 1-band tightbinding basis which can be used to calculated the bulk band structure for the $n$th band. However, the interaction among those functions will in principle extend to large distances, which makes computations inefficient. The Generalized Planar Wannier Functions (GPWF) can be used to solve this problem. The generalized planar Wannier functions are proper linear combinations of $W_{n, k \|}\left(R_{z}\right)$ for the interested bands such that they are more localized along the $z$ direction. In this new sub-basis, the interaction parameters can be cut off at shorter distances, but at the cost of leaving the Hamiltonian in the GPWF sub-basis non-diagonal in band index. In addition, depending on the number of neighboring interactions kept, the GPWF sub-basis could be either orthogonal or non-orthogonal.

For the generalized planar Wannier functions, the elements of the Hamiltonian matrix are given by,

$$
H_{m, m^{\prime}}(\mathbf{k})=\sum_{R_{z}} e^{i k_{z} R_{z}}\left\langle W_{m, \mathbf{k}_{\|}}^{\prime}(0)|H| W_{m^{\prime}, \mathbf{k}_{\|}}^{\prime}\left(R_{z}\right)\right\rangle
$$

where $W_{m^{\prime}, \boldsymbol{k}_{\|}}^{\prime}$ is a generalized planar Wannier function. By storing the interaction terms $\left\langle W_{m, \boldsymbol{k}_{\|}}^{\prime}(0)|H| W_{m^{\prime}, \boldsymbol{k}_{\|}}^{\prime}\left(R_{z}\right)\right\rangle$ up to the desired number of neighbors (two for non-orthogonal, and four for orthogonal basis) for each fixed value of $\boldsymbol{k}_{\|}$, we can converted the problem into a 1D multiband tight-binding model.

Let $d$ be monolayer separation along the growth direction. We can then write $R_{z}=\sigma \mathrm{d}$, where $\sigma$ is a integer layer label. We can denote the generalized Wannier planar functions with the following tightbinding-like simplified notation :

$$
\left|\sigma, \mathbf{k}_{\|}\right\rangle \equiv\left|W_{m, \mathbf{k}_{\|}}^{\prime}\left(R_{z}=\sigma d\right)\right\rangle
$$

where $m=1,2, \ldots, M$ labels the basis functions within a monolayer ( $M=16$ in our case). We then appeal to MQTBM for transmission coefficient calculations. 


\section{B. Transmission Coefficient Calculations in Non-Orthogonal Basis}

The multiband quantum transmitting boundary method is a numerically stable and efficient formulation for calculating heterostructure transmission coefficients using realistic multiband tightbinding band structure model. As seen in the previous section, the Generalized Planar Wannier Functions (GPWF) may be viewed as a nonorthogonal tight-binding basis with second-neighbor interactions. Previously, MQTBM has been implemented for several nearest-neighbor tightbinding models with orthogonal bases. The generalization to orthogonal basis with multiple neighbor interactions is straight-forward. However, a somewhat different formulation is required for non-orthogonal bases. In this work, we will introduce a more general version of MQTBM capable of handling multiple-neighbor interactions in a non-orthogonal basis. We consider a heterostructure as consisting of an active region sandwiched between two semi-infinite flat-band electrode regions. The computational domain consists of a sequence of $N$ monolayers parallel to heterointerfaces. It includes all of the active region, and extends out from both sides of the active region to include $2 N_{I}$ layers of each of the flat-band electrodes; $N_{I}$ is the number of neighboring interactions for the particular tight-binding model used. As will be been, these inclusions are sufficient to allow us to fully incorporate the scattering boundary conditions within the finite computational domain.

We label the layers in the computational domain by $\sigma=1,2, \ldots, N$. For a second-neighbor model, for example, $\sigma=1,2,3,4$ will be in the left electrode, $\sigma=N-3, N-2, N-1, N$ the right and the rest are the active layer. From this point on we will specifically examine the case of a secondneighbor model for simplicity. However, all derivations generalize trivially to models with larger interaction range.

A wave function in the tight-binding basis may be written as

$$
|\Psi\rangle=\sum_{\sigma m} C_{\sigma m}\left|\sigma m, \boldsymbol{k}_{\|}\right\rangle
$$

where, again, $\boldsymbol{k}_{\|}$is the planar orbital in-plane wave vector, $\sigma$ the layer label and $m$ the orbital label.

The Schrödinger equation $(H-E)|\Psi\rangle=0$ in this basis may be written as

$$
\begin{aligned}
\overline{\boldsymbol{H}}_{\sigma, \sigma-2} \boldsymbol{C}_{\sigma-2} & +\overline{\boldsymbol{H}}_{\sigma, \sigma-1} \boldsymbol{C}_{\sigma-1}+\overline{\boldsymbol{H}}_{\sigma, \sigma} \boldsymbol{C}_{\sigma} \\
& +\overline{\boldsymbol{H}}_{\sigma, \sigma+1} \boldsymbol{C}_{\sigma+1}+\overline{\boldsymbol{H}}_{\sigma, \sigma+2} \boldsymbol{C}_{\sigma+2}=0
\end{aligned}
$$

where $C_{\sigma}$ is a vector of length $M$,

$$
\boldsymbol{C}_{\sigma}=\left(\begin{array}{c}
C_{\sigma 1} \\
C_{\sigma 2} \\
\vdots \\
C_{\sigma M}
\end{array}\right)
$$

and $\overline{\boldsymbol{H}}_{\sigma, \sigma^{\prime}}$ are $M \times M$ matrices,

$$
\overline{\boldsymbol{H}}_{\sigma, \sigma^{\prime}}=\boldsymbol{H}_{\sigma, \sigma^{\prime}}-E \boldsymbol{S}_{\sigma, \sigma^{\prime}}
$$

The elements of the Hamiltonian matrix $\boldsymbol{H}$ and overlap matrix $\boldsymbol{S}$ are given, respectively, by :

$$
\left(\boldsymbol{H}_{\sigma, \sigma^{\prime}}\right)_{m, m^{\prime}}=\left\langle\sigma m, \boldsymbol{k}_{\|}|H| \sigma^{\prime} m^{\prime}, \boldsymbol{k}_{\|}\right\rangle
$$

and

$$
\left(\boldsymbol{S}_{\sigma, \sigma^{\prime}}\right)_{m, m^{\prime}}=\left\langle\sigma m, \boldsymbol{k}_{\|} \mid \sigma^{\prime} m^{\prime}, \boldsymbol{k}_{\|}\right\rangle
$$

For convenience, we do not explicitly show the dependence on $\boldsymbol{k}_{\|}$and $E$ in our notation.

The boundary conditions in scattering problems are usually described in terms of Bloch states, i.e., as amplitudes of incoming and outgoing plane waves. To translate them into the tight-binding basis, we make use of the transfer matrix and Bloch's theorem. Together, they provide a connection between the plane-wave and the local-orbital descriptions through the concept of complex band structures [7]. 
We can write Eq. 5 in the transfer-matrix form,

$$
\left(\begin{array}{cccc}
-\overline{\boldsymbol{H}}_{\sigma, \sigma-2}^{-1} \overline{\boldsymbol{H}}_{\sigma, \sigma-1} & -\overline{\boldsymbol{H}}_{\sigma, \sigma-2}^{-1} \overline{\boldsymbol{H}}_{\sigma, \sigma} & -\overline{\boldsymbol{H}}_{\sigma, \sigma-2}^{-1} \overline{\boldsymbol{H}}_{\sigma, \sigma+1} & -\overline{\boldsymbol{H}}_{\sigma, \sigma-2}^{-1} \overline{\boldsymbol{H}}_{\sigma, \sigma+2} \\
\boldsymbol{I} & 0 & 0 & 0 \\
0 & \boldsymbol{I} & 0 & 0 \\
0 & 0 & \boldsymbol{I} & 0
\end{array}\right)\left(\begin{array}{c}
\boldsymbol{C}_{\sigma-1} \\
\boldsymbol{C}_{\sigma} \\
\boldsymbol{C}_{\sigma+1} \\
\boldsymbol{C}_{\sigma+2}
\end{array}\right)=\left(\begin{array}{c}
\boldsymbol{C}_{\sigma-2} \\
\boldsymbol{C}_{\sigma-1} \\
\boldsymbol{C}_{\sigma} \\
\boldsymbol{C}_{\sigma+1}
\end{array}\right)
$$

Now, consider, plane-wave state in either of the flat-band electrode regions,

$$
\left.\left|\psi_{I}(\boldsymbol{k}\rangle=\right| \psi_{I}\left(\boldsymbol{k}_{\|}, \boldsymbol{k}_{\perp}\right)\right\rangle=\sum_{\sigma m} \boldsymbol{B}_{\sigma m}\left|\sigma m, \boldsymbol{k}_{\|}\right\rangle .
$$

Since $\mid \psi_{I}(\boldsymbol{k}\rangle$ is a Bloch state, by Bloch's theorem, the tight-binding coefficients must obey the relation

$$
\boldsymbol{B}_{\sigma}=e^{i k_{\perp} d} \boldsymbol{B}_{\sigma-1},
$$

where $d$ is the distance between monolayers, and $k_{\perp}$ is the component of the crystal momentum along the growth direction. Then, together with Eq. (10), we can formulate the following eigenvalue problem: binding basis and Bloch (plane-wave) states [4]. We sort the eigenvectors such that the first $2 M$ vectors represent state which propagate or decay to the right, and the remainder $2 M$ to the left. The sorted eigenvectors are then grouped in a $4 \times 4 M$ matrix $\boldsymbol{D}$.

Assume that we have a known coefficient set of incoming plane wave, $\tilde{\boldsymbol{I}}$, and unknown coefficient sets of reflected and transmitted parts, $\tilde{\boldsymbol{r}}$ and $\tilde{\boldsymbol{t}}$, where $\tilde{\boldsymbol{I}}, \tilde{\boldsymbol{r}}$ and $\tilde{\boldsymbol{t}}$ are the column vectors of the length $2 M$. Then we can find the relations:

$$
\left(\begin{array}{l}
\tilde{\boldsymbol{C}}_{1} \\
\tilde{\boldsymbol{C}}_{2}
\end{array}\right)=\boldsymbol{D}^{L}\left(\begin{array}{c}
\tilde{\boldsymbol{I}} \\
\tilde{\boldsymbol{r}}
\end{array}\right)=\left(\begin{array}{ll}
\boldsymbol{D}_{11}^{L} & \boldsymbol{D}_{12}^{L} \\
\boldsymbol{D}_{21}^{L} & \boldsymbol{D}_{22}^{L}
\end{array}\right)\left(\begin{array}{c}
\tilde{\boldsymbol{I}} \\
\tilde{\boldsymbol{r}}
\end{array}\right),
$$

$$
\left(\begin{array}{cccc}
-\overline{\boldsymbol{H}}_{\sigma, \sigma-2}^{-1} \overline{\boldsymbol{H}}_{\sigma, \sigma-1} & -\overline{\boldsymbol{H}}_{\sigma, \sigma-2}^{-1} \overline{\boldsymbol{H}}_{\sigma, \sigma} & -\overline{\boldsymbol{H}}_{\sigma, \sigma-2}^{-1} \overline{\boldsymbol{H}}_{\sigma, \sigma+1} & -\overline{\boldsymbol{H}}_{\sigma, \sigma-2}^{-1} \overline{\boldsymbol{H}}_{\sigma, \sigma+2} \\
1 & 0 & 0 & 0 \\
0 & 1 & 0 & 0 \\
0 & 0 & 1 & 0
\end{array}\right)\left(\begin{array}{c}
\boldsymbol{B}_{\sigma-1} \\
\boldsymbol{B}_{\sigma} \\
\boldsymbol{B}_{\sigma+1} \\
\boldsymbol{B}_{\sigma+2}
\end{array}\right)=e^{i k \perp d}\left(\begin{array}{c}
\boldsymbol{B}_{\sigma-1} \\
\boldsymbol{B}_{\sigma} \\
\boldsymbol{B}_{\sigma+1} \\
\boldsymbol{B}_{\sigma+2}
\end{array}\right)
$$

Recall the implicit dependence of $\overline{\boldsymbol{H}}_{\sigma, \sigma^{\prime}}$ on $E$. Solving the above yields a set of eigenvalues $\left\{e^{i k \perp d}\right\}$, from which a corresponding set of complex $\left\{k_{\perp}\right\}$ values are readily calculated. $E\left(k_{\perp}\right)$ is the bulk complex band structure [7]. As an example, the complex band structure for bulk GaAs calculated using the planar-basis pseudopotential band structure model is shown in Figure 1.

Each of the $4 M$ eigenvectors contain the coefficients of tight-binding orbitals on the one hand, and is associated with a particular complex wave vector $(\boldsymbol{k} \|, \boldsymbol{k} \perp)$ on the other. In this way, they provide a connection between the local tight- and

$$
\left(\begin{array}{c}
\tilde{\boldsymbol{C}}_{(N / 2)-1} \\
\tilde{\boldsymbol{C}}_{N / 2}
\end{array}\right)=\boldsymbol{D}^{R}\left(\begin{array}{c}
\tilde{\boldsymbol{t}} \\
0
\end{array}\right)=\left(\begin{array}{ll}
\boldsymbol{D}_{11}^{R} & \boldsymbol{D}_{12}^{R} \\
\boldsymbol{D}_{21}^{R} & \boldsymbol{D}_{22}^{R}
\end{array}\right)\left(\begin{array}{c}
\tilde{\boldsymbol{t}} \\
\boldsymbol{0}
\end{array}\right),
$$

where $D^{L}$ and $D^{R}$ are $4 M \times 4 M$ matrices formed by the eigenvectors obtained by solving Eq. (13), for the bulk materials making up the left and right electrodes, respectively, and the $\tilde{\boldsymbol{C}}_{j}$ is a vector of length $2 M$ constructed by joining two consecutive $\boldsymbol{C}$ vectors : $\tilde{\boldsymbol{C}}_{1}$ contain $\boldsymbol{C}_{1}$ and $\boldsymbol{C}_{2}, \tilde{\boldsymbol{C}}_{2}$ contain $\boldsymbol{C}_{3}$ and $\boldsymbol{C}_{4}, \tilde{\boldsymbol{C}}_{(N / 2)-1}$ contain $\boldsymbol{C}_{N-3}$ and $\boldsymbol{C}_{N-2}$ and $\tilde{\boldsymbol{C}}_{N / 2}$ 
contain $C_{N-1}$ and $C_{N}$ instead. Note that we have divided each $D_{i j}$ matrix into $2 M \times 2 M$ sub-matrices for convenience.

Eliminating $\tilde{\boldsymbol{r}}$ and $\tilde{\boldsymbol{t}}$ from Eqs. (14) and (15), we obtain

$$
\tilde{\boldsymbol{C}}_{1}-\boldsymbol{D}_{12}^{L} \boldsymbol{D}_{22}^{L-1} \tilde{\boldsymbol{C}}_{2}=\boldsymbol{D}_{11}^{L} \tilde{\boldsymbol{I}}-\boldsymbol{D}_{11}^{L} \boldsymbol{D}_{22}^{L-1} \boldsymbol{D}_{21}^{L} \tilde{\boldsymbol{I}}
$$

and

$$
-\boldsymbol{D}_{21}^{R} \boldsymbol{D}_{11}^{R-1} \tilde{\boldsymbol{C}}_{(N / 2)-1}+\tilde{\boldsymbol{C}}_{N / 2}=0
$$

Combining the above equations and Eq. (5), we obtain $M N$ linear equations written in the matrix form as

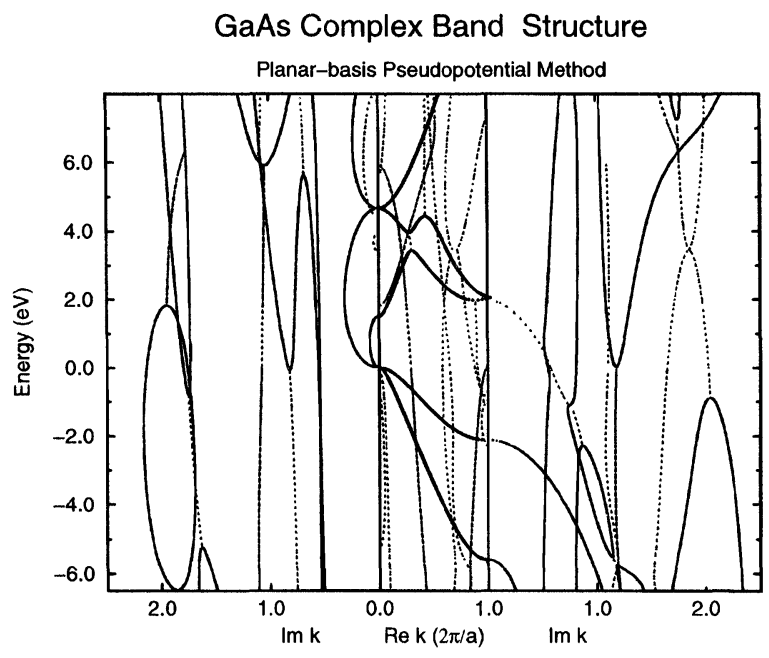

FIGURE 1 Complex bands of GaAs calculated using the planar-basis pseudopotential method.

$$
\begin{gathered}
\left(\begin{array}{ccccccccc}
I_{M} & 0 & {\left[-D_{12}^{L} D_{22}^{L}{ }^{-1}\right]} & 0 & \cdots & \cdots & \cdots & 0 \\
0 & I_{M} & & \cdots & \cdots & \cdots & 0 \\
\bar{H}_{31} & \bar{H}_{32} & \bar{H}_{33} & \bar{H}_{34} & \bar{H}_{35} & 0 & \cdots & \cdots & 0 \\
0 & \bar{H}_{42} & \bar{H}_{43} & \bar{H}_{44} & \bar{H}_{45} & \bar{H}_{46} & 0 & \vdots & \vdots \\
\vdots & \ddots & \ddots & \ddots & \vdots & \vdots & \vdots & \vdots & \vdots \\
0 & \cdots & \cdots & \cdots & 0 & {\left[-D_{21}^{R} D_{11}^{R-1}\right]} & I_{M} & 0 \\
0 & \cdots & \cdots & \cdots & I_{M}
\end{array}\right)\left(\begin{array}{c}
C_{1} \\
C_{2} \\
C_{3} \\
C_{4} \\
\vdots \\
C_{N-1} \\
C_{N}
\end{array}\right) \\
=\left(\begin{array}{c}
{\left[D_{11}^{L} \tilde{\boldsymbol{I}}-D_{12}^{L} D_{22}^{L}{ }^{-1} D_{21}^{L} \tilde{\boldsymbol{I}}\right.} \\
0 \\
0 \\
0 \\
0 \\
0 \\
0 \\
0
\end{array}\right)
\end{gathered}
$$

where $I_{M}$ is an $M \times M$ identity matrix and the bracketed terms are $2 M \times 2 M$ sub-matrices. Solving the matrix equation yields a set of coefficient, $\left\{\boldsymbol{C}_{1}, \boldsymbol{C}_{2}, \ldots, \boldsymbol{C}_{N}\right\}$. By Eq. (15), the coefficients of the transmitted plane-wave states can be computed by

$$
\tilde{\boldsymbol{t}}=\boldsymbol{D}_{21}^{R-1} \boldsymbol{C}_{N / 2}
$$

We can use $\tilde{\boldsymbol{t}}$ to compute the transmission coefficient :where $v_{I}\left(E, k_{\|} ; L\right)$ and $v_{j}\left(E, k_{\|} ; R\right)$ are the velocities of the incident and the transmitted bulk plane-waves, respectively.

\section{RESULTS AND DISCUSSION}

We have successfully applied the multiband quantum transmitting boundary method to tightbinding band structure models with nonorthogonal basis and multiple-neighbor interactions. 
Figure 2 shows transmission spectra of two different GaAs/AlAs double barrier structures calculated for using the Generalized Planar Wannier Functions (GPWF) band-structure model with second-neighbor interactions. Both $\Gamma$ - point and the $X$-point resonant tunneling are seen in Figure 2. The $\Gamma$ - and $X$-point resonances are associated with quasibound states localized in the GaAs and the AlAs layers respectively. The two structures used have the same GaAs layer widths (16 monolayers) but different AlAs layer widths (4 and 8 monolayers). Consequently, the positions of the $\Gamma$-point resonances remain the same for the two structures, while the $X$-point resonances showed a definite position shift.

\section{Acknowledgment}

This work was supported by the ROC National Science Council under Grant No. NSC 86-2112-M007-001, and by the US Office of Naval Research under contract No. ONR-N00014-90-J-1267. The use of ROC National Center for High-Performance Computing facilities is acknowledged.

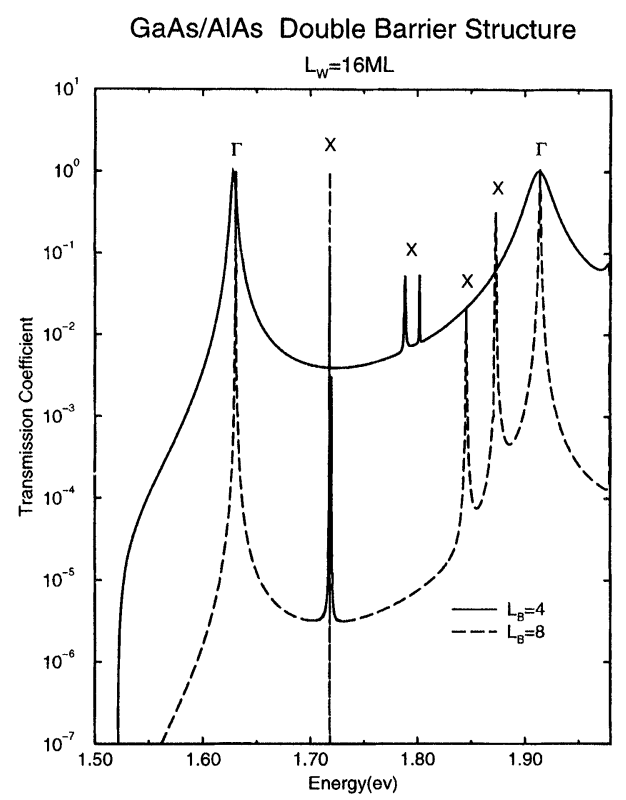

FIGURE 2 Transmission coefficients of the GaAs/AlAs double barrier structure.

\section{References}

[1] Chang, L. L., Esaki, L. and Tsu, R. (1974). "Resonant tunneling in semiconductor double barrier", Appl. Phys. Letts., 24(12), 593.

[2] Ting, D. Z.-Y., Yu, E. T. and McGill, T. C. (1992). "Multiband treatment of quantum transport in interband tunnel devices", Phys. Rev. B., 45(7), 3583.

[3] Lent, C. S. and Kirkner., D. J. (1990). "The quantum transmitting boundary method", J. Appl. Phys., 67, 6353.

[4] Ting, D. Z.-Y. and McGill, T. C. "Multiband and Multidimensional Analysis of Quantum Transport in Ultrasubmicron Device", published in Quantum Transport Ultrasmall in Device, edit by D. K. Ferry, H. L. Grubin, C. Jacoboni and A.-P. Jauho, NATO ASI Series, Series B : Physic; 342, (Plenum Press, New York, 1995), pp. 417-436.

[5] Chang, Y.-C. (1995). "Device modeling from first principles at the atomic level", Proceedings of the 2nd international symposium on compound semiconductors, Cheju Island, Korea.

[6] Chang, Y.-C. and Li, G. (1996). "Planar-basis pseudopotential method and planar Wannier functions for surfaces and heterostructures", Comp. Phys. Commun., 95(2-3), 158.

[7] Chang, Y.-C. and Schulman, J. N. (1983). "Complex band structures of crystalline solids", Phys. Rev. B., 27, 2346.

\section{Authors' Biographies}

Geng-Chiau Liang received a B.S. degree (1995) and an M.S. degree (1997) in Physics from the National Tsing Hua University, Taiwan. His research involves theoretical studies of transport in semiconductor heterostructures.

Yiping A. Lin received a B.S. degree (1995) and an M.S. degree (1997) in Physics from the National Tsing Hua University, Taiwan. Her research interests include modeling of scanning probe microscopy and semiconductor physics.

David Z.-Y. Ting is an Associate Professor of Physics at the the National Tsing Hua University in Hsinchu, Taiwan, ROC, and a Visiting Research Associate at the California Institute of Technology. His research activities include theoretical studies of electronic and optical properties of semiconductor alloys and heterostructures, quantum transport in nanostructures and tunnel devices, and optical simulations.

Yia-Chung Chang is a Professor of Physics at the University of Illinois at Urbana-Champaign. His research interests include shallow impurities and excitons bound to shallow impurities in semicon- 
ductors, shallow impurities in semiconductor quantum wells, many body effects in semiconductors with high carrier densities, exciton dynamics, electronic and optical properties of superlattices, magnetotransport and exchange interactions in magnetic multilayers, optical properties of disor- dered semiconductors, electronic properties of semiconductor surfaces, nonlinear optical properties of semiconductors, impact ionization in semiconductors, and physics of heterostructure resonant tunneling devices, nanostructure lasers, and infrared detectors. 

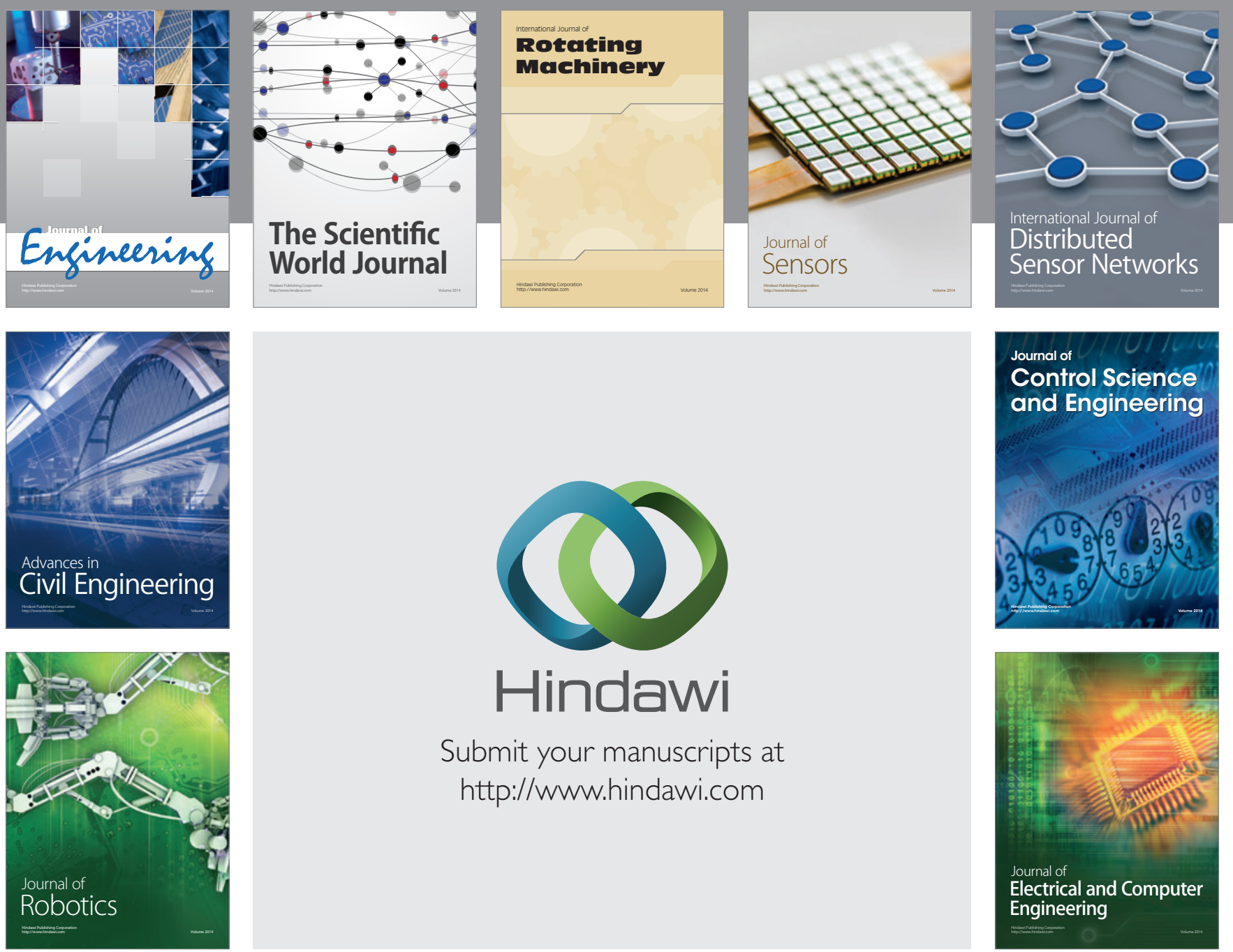

Submit your manuscripts at

http://www.hindawi.com
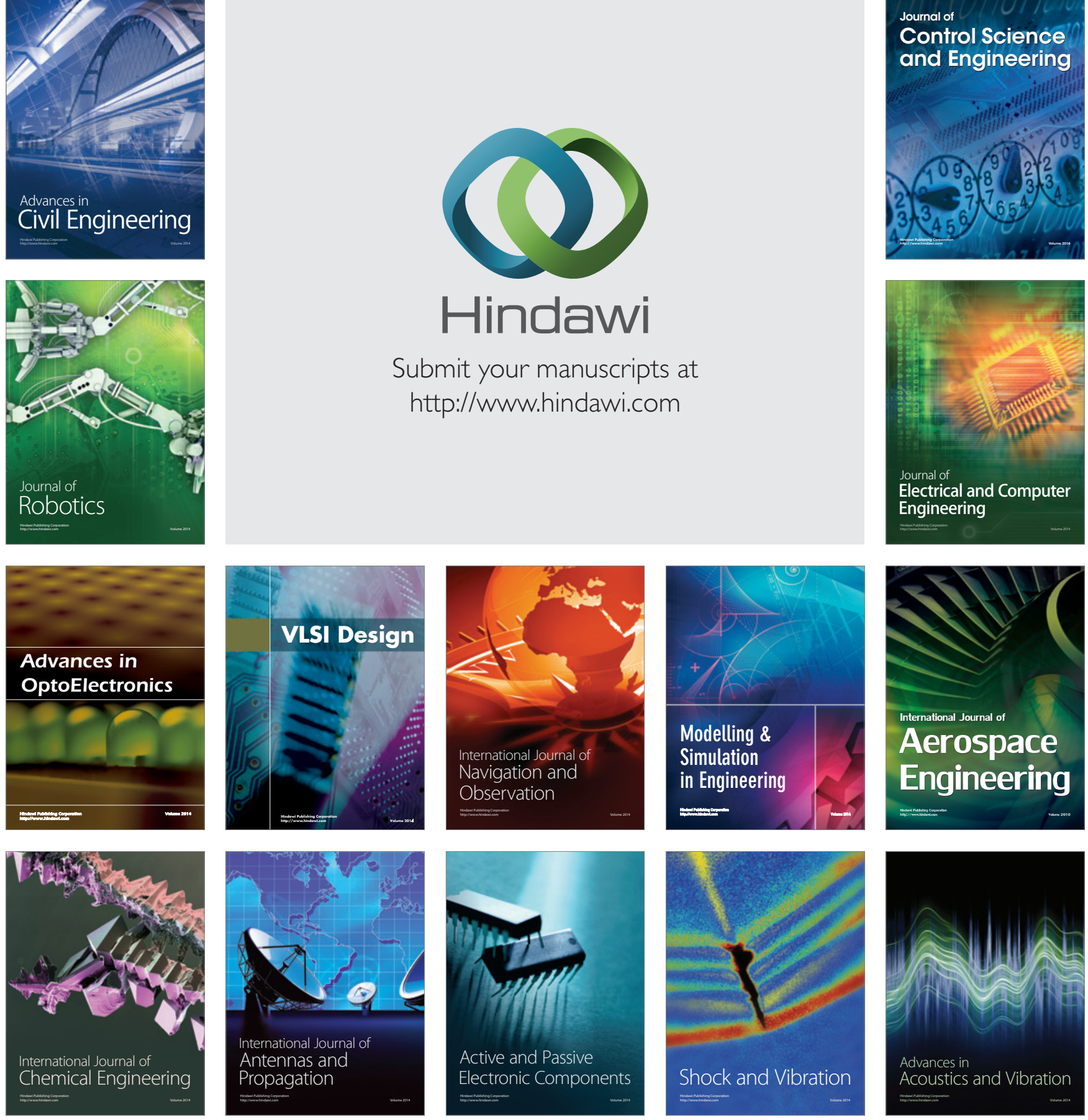